\title{
Decoupling can revive minimal supersymmetric SU(5)
}

\section{Takumi Kuwahara*}

Department of Physics, Nagoya University, Nagoya 464-8602, Japan

E-mail: kuwaharaeth.phys.nagova-u.ac.jp

We study the proton lifetime in the minimal SUSY SU(5) grand unified theory (GUT) with the high scale SUSY breaking mechanism. In the minimal SUSY SU(5) GUT, which is the minimal GUT extension of the SM, the partial lifetime of proton for the mode $p \rightarrow K^{+}+\bar{v}$ is predicted about $10^{30}$ years. On the other hand, an experimental lower bound for this mode is $5.9 \times 10^{33}$ years. Since the theoretical prediction is in contradiction with this experimental results, the dimension-five effective operators which give rise to this decay must be suppressed or forbidden by some mechanism. The high-scale SUSY breaking mechanism gains attention as the most simple SUSY model which explains the recent SUSY search experiments at collider and the mass of the SM Higgs boson. As a results, we have revealed that the dimension-five operators are not need to suppress or forbid; this decay mode may be discovered in the future experiment at Hyper-Kamiokande.

KMI International Symposium 2013 on "Quest for the Origin of Particles and the Universe", 11-13 December, 2013

Nagoya University, Japan

\footnotetext{
* Speaker.
} 


\section{Introduction}

In this paper, we briefly summarize our analysis of the partial proton lifetime for the decay mode $p \rightarrow K^{+}+\bar{v}$ in the minimal supersymmetric SU(5) grand unified theory (SUSY SU(5) GUT) with the high-scale SUSY breaking mechanism [四].

The Large Hadron Collider (LHC) experiments give us various information; the discovery of the $126 \mathrm{GeV}$ Higgs boson [ [ [ ] ] and the severe constraint on the masses of the superpartners with the color charge. In the minimal supersymmetric standard model (MSSM) with the low scale SUSY breaking whose typical energy scale is around TeV, the Higgs boson mass is lighter than the mass of the Z-boson at tree-level. Lately, the high-scale SUSY scenarios have gotten a lot of attention recently since the observed mass of the Higgs boson is realized by large quantum corrections. In this scenario, we assume that there is no gauge singlet field in the SUSY breaking sector. The soft masses of squarks, sleptons, and heavy Higgs are generated by the higher dimensional operators. On the other hand, the leading contribution to the gaugino mass terms and the A-terms are generated through the anomaly-mediated SUSY breaking mechanism [四, [] since the gauge invariant higher dimensional operators are forbidden. In particular, the gaugino mass $M_{a}$ is obtained as follows:

$$
M_{a}=\frac{b_{a} g_{a}^{2}}{16 \pi^{2}} m_{3 / 2}
$$

where $g_{a}$ and $b_{a}$ are the gauge coupling and the coefficient of the 1-loop $\beta$ functions of the gauge coupling, respectively. $m_{3 / 2}$ denotes the gravitino mass.

The minimal SUSY SU(5) GUT is the most minimal extended theory of the standard model. It is well known that the minimal SUSY SU(5) GUT has been excluded due to predicting too short proton lifetime $[$ 团, 四]. This short lifetime is given rise to by the color-triplet Higgs multiplets which are the SU(5) partners of the MSSM Higgs doublets: the main decay mode is proton to charged $\mathrm{K}$ meson and anti-neutrino. Thus, the interactions which give rise to the terrible nucleon decay have to be suppressed or forbidden by some mechanism.

\section{The minimal SUSY SU(5) GUT}

The MSSM superfields are embedded in the SU(5) multiplets. First, the left-handed quark doublet $Q^{a r}$, the charge conjugated right-handed up-type quark $U_{a}^{C}$, and the charge conjugated right-handed charged lepton $E^{C}$ are embedded in the anti-symmetric representation $\Psi$. The other MSSM chiral superfields, the charge-conjugated right-handed down-type quark $D_{a}^{C}$ and the lefthanded lepton doublet $L^{s}$, are embedded into the anti-fundamental representation $\Phi$ :

$$
\Psi^{\alpha \beta}(\mathbf{1 0})=\frac{1}{\sqrt{2}}\left(\begin{array}{cc}
\varepsilon^{a b c} U_{c}^{C} & Q^{a r} \\
-Q^{s a} & \varepsilon^{s r} E^{C}
\end{array}\right), \Phi_{\alpha}(\overline{\mathbf{5}})=\left(\begin{array}{c}
D_{a}^{C} \\
\varepsilon_{r s} L^{s}
\end{array}\right),
$$

$\alpha, \beta, \cdots=1,2, \cdots 5$ represent the $S U(5)$ indices. The roman indices $(a, b, c, \cdots=1,2,3)$ denote the $S U(3)_{C}$ indices and the roman indices $(r, s, \cdots=1,2)$ denote the $S U(2)_{L}$ indices.

Two Higgs doublets in MSSM, $H_{u}$ and $H_{d}$, are embedded in a pair of $\mathbf{5}$ and $\overline{\mathbf{5}}$ representation chiral superfields with the color-triplet Higgs multiplets $H_{C}$ and $\bar{H}_{C}$.

$$
H(\mathbf{5})=\left(\begin{array}{c}
H_{C}^{a} \\
H_{u}^{r}
\end{array}\right), \quad \bar{H}(\overline{\mathbf{5}})=\left(\begin{array}{c}
\bar{H}_{C a} \\
\varepsilon_{r s} H_{d}^{s}
\end{array}\right) .
$$


These color-triplet Higgs multiplets give rise to the terrible proton decay. The dimension-five operators are generated by integrating out the massive color-triplet Higgs multiplets since the mass term of the color-triplet Higgs is obtained as $M_{H_{C}} H_{C} \bar{H}_{C}$ after the GUT breaking.

Since these operators generate the two-Fermion and two-Sfermion vertices, we obtain the four-Fermi operators by mediating the superpartners of the charged Higgs bosons or the charged W bosons (Fig.W). The main decay mode caused by the dimension-five operators is $p \rightarrow K^{+}+\bar{v}$ due to the totally anti-symmetric tensor of $S U(3)_{C}$ in the Wilson coefficients of these operators. The amplitudes for this decay mode are proportional to the mass function $F\left(\mu, M_{S}\right)$ defined as:

$$
F\left(\mu, M_{S}\right) \equiv \frac{\mu}{M_{S}^{2}-\mu^{2}}\left(1-\frac{\mu^{2}}{M_{S}^{2}-\mu^{2}} \ln \frac{M_{S}^{2}}{\mu^{2}}\right) \sim \frac{\mu}{M_{S}^{2}},
$$

where $\mu$ is the mass of the charged wino or the charged higgsino and $M_{S}$ denotes the mass of the degenerate sfermions. In particular, in the case that the higgsino is degenerate in mass $M_{S}$, we obtain the partial proton lifetime for this decay mode approximately as follows:

$$
\tau_{p} \sim\left(4.0 \times 10^{35}\right) \times \sin ^{4} 2 \beta\left(\frac{0.1}{A_{R}}\right)^{2}\left(\frac{M_{S}}{10^{2} \mathrm{TeV}}\right)^{2}\left(\frac{M_{H_{C}}}{10^{16} \mathrm{GeV}}\right)^{2} \quad[\text { years] },
$$

where $\tan \beta$ is the ratio of the vacuum expectation values of the MSSM Higgs multiplets and $A_{R}$ denotes the renormalization factor of the Wilsonian coefficients of the dimension-five operators, the Yukawa couplings, and so on.

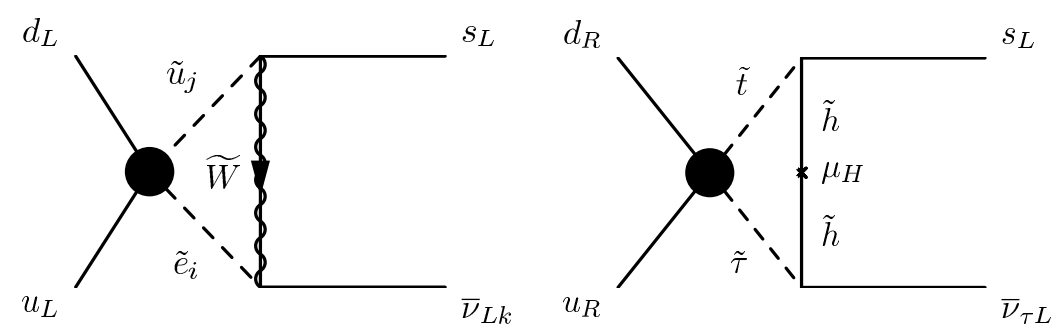

Figure 1: The four-Fermi operators which give rise to proton decay are induced by mediating the sparticles. Black dots in these figures display the dimension-five operators.

\section{Proton Decay with High-Scale SUSY}

In our analysis, we assume that squarks, sleptons, and heavy Higgs bosons are degenerate in mass $\left(M_{S}\right)$. We also assume that the mass scale of the color-triplet Higgs multiplets are set to be $10^{16} \mathrm{GeV}$ since the color-triplet Higgs multiplets are able to have the heavy mass in the high-scale SUSY scenario [ [8]].

In Fig. 凹, we show that the theoretical prediction on this partial proton lifetime is consistent with the experimental lower bound in the broad parameter region [四]. In particular, the mass of the observed Higgs boson is realized in a parameter set $\left(\tan \beta \approx 2 \sim 3, M_{S} \approx 10^{2} \mathrm{TeV}\right)$. Furthermore, we may discover this decay mode in the future Hyper-Kamiokande experiment since the lower bound on this decay mode achieves $2.5 \times 10^{34}$ years. 

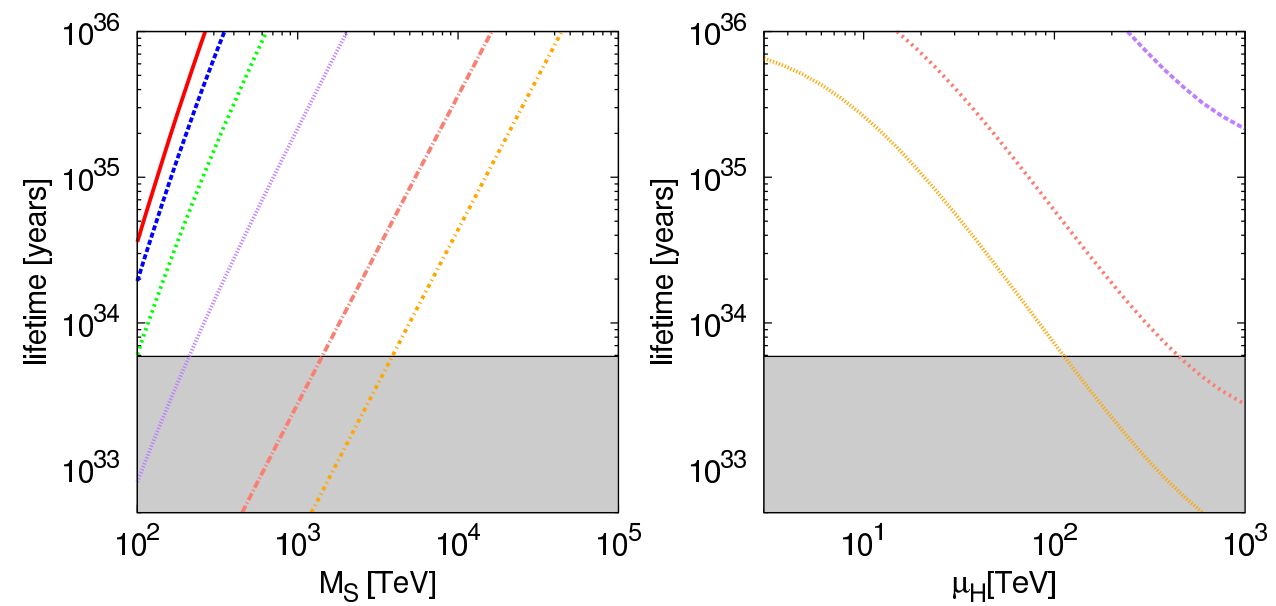

Figure 2: (Left figure): In this figure, we have evaluated the partial proton lifetime in the case that the higgsino is degenerate in squark mass $\left(M_{S}\right)$. From left-top to right-bottom, these lines correspond to $\tan \beta=2,3,5,10,30$, and 50. The wino mass $\left(M_{2}\right)$ are set to be $3 \mathrm{TeV}$. (Right figure): In this figure, we have evaluated the partial proton lifetime as a function of the mass of the higgsino $\mu_{H}$. From right-top to left-bottom, these lines correspond to $\tan \beta=10,30$, and 50 in this figure. The wino mass $\left(M_{2}\right)$ and the sfermion mass $\left(M_{S}\right)$ are set to be $3 \mathrm{TeV}$ and $10^{3} \mathrm{TeV}$, respectively. The gray region is excluded by the Super-Kamiokande experiment $\left(\tau>5.9 \times 10^{33}\right.$ years [Q] $)$ in each figure.

\section{Summary}

We have evaluated the partial proton lifetime for the decay mode $\left(p \rightarrow K^{+}+\bar{v}\right)$ via the color-triplet Higgs multiplets in the high-scale SUSY scenario. The theoretical prediction on this decay mode in this scenario can be evaded from the experimental lower bound $\left(\tau_{p}>5.9 \times 10^{33}\right.$ years). Our results also indicate that this decay mode may be discovered in the future Hyper-K experiment. In the point of view of the model buildings, the additional symmetries which suppress or prohibit the vector-like mass term $M_{H_{C}} H_{C} \bar{H}_{C}$ or the dimension-five operators are not needed in the high-scale SUSY breaking scenario.

\section{References}

[1] J. Hisano, D. Kobayashi, T. Kuwahara and N. Nagata, JHEP 1307 (2013) 038.

[2] S. Chatrchyan et al. [CMS Collaboration], Phys. Lett. B 716 (2012) 30 .

[3] G. Aad et al. [ATLAS Collaboration], Phys. Lett. B 716 (2012) 1 .

[4] L. Randall and R. Sundrum, Nucl. Phys. B 557 (1999) 79.

[5] G. F. Giudice, M. A. Luty, H. Murayama and R. Rattazzi, JHEP 9812 (1998) 027.

[6] T. Goto and T. Nihei, Phys. Rev. D 59 (1999) 115009.

[7] H. Murayama and A. Pierce, Phys. Rev. D 65 (2002) 055009.

[8] J. Hisano, T. Kuwahara and N. Nagata, Phys. Lett. B 723 (2013) 324 .

[9] M. Shiozawa, Preliminary results for the Super-Kamiokande Collaboration, presented at TAUP 2013, Asilomar, CA. 